\title{
Monte Carlo simulation and reference hypernetted chain equation results for structural, thermodynamic, and dielectric properties of polar heteronuclear diatomic fluids
}

\author{
M. Lombardero and C. Martín \\ Instituto de Química Física Rocasolano, CSIC, Serrano 119, E-28006 Madrid, Spain \\ and Departamento de Química Física I, U. Complutense, E-28040 Madrid, Spain \\ E. Lomba \\ Instituto de Química Física Rocasolano, CSIC, Serrano 119, E-28006 Madrid, Spain \\ F. Lado \\ Department of Physics, North Carolina State University, Raleigh, North Carolina 27695
}

(Received 14 September 1995; accepted 8 November 1995)

\begin{abstract}
We study fluids of heteronuclear two-center Lennard-Jones molecules with embedded point dipoles using both numerical simulation and integral equation theory. Extensive Monte Carlo simulations are performed for the structural, thermodynamic, and dielectric properties of two models of such fluids, with unusually long simulation runs to assure convergence of the dielectric constant. The results are used to test a generalization of a reference hypernetted chain approximation (RHNC-VM) used previously for nonpolar heteronuclear diatomics. Very good agreement is found between the two methods. We conclude that the RHNC-VM integral equation is a reliable method for studying both polar and nonpolar fluids of diatomic molecules. (C) 1996 American Institute of Physics. [S0021-9606(96)01507-X]
\end{abstract}

\section{INTRODUCTION}

In a recent paper, ${ }^{1}$ we have applied, with good results, a reference hypernetted chain (RHNC) approximation to fluids of uncharged heteronuclear diatomic molecules with sitesite Lennard-Jones interactions. This approximation, denoted RHNC-VM, which has proven to be equally successful for homonuclear systems with and without quadrupolar interactions (see Ref. 1 and references therein) takes, as a reference system, a fluid of hard diatomics whose atomic diameters, $d_{a}$ and $d_{b}$, depend on the temperature $T$ and the density $\rho$ of the fluid of interest according to the empirical relation ${ }^{1}$

$$
d_{\alpha}=\sigma_{\alpha} g(\gamma)\left[25-\ln \left(k T / \epsilon_{\alpha}\right)\right]\left[25+\ln \left(\rho \sigma_{\alpha}^{3}\right)\right], \quad \alpha=a, b
$$

with

$$
g(\gamma)=\alpha\left[1-h\left(\frac{1-\gamma}{1+\gamma}\right)^{2}\right]
$$

where $\gamma=\sigma_{b} / \sigma_{a} \leqslant 1, \epsilon_{\alpha}, \sigma_{\alpha}$ being the Lennard-Jones parameters for the $\alpha \alpha$ atomic interactions, and in Eq. (2) $\alpha=1.62 \times 10^{-3}$ and $h=0.98$ (Ref. 2) are adjustable parameters. Their values have been determined by requiring that, for some convenient thermodynamic state at high density, the computed values of the principal thermodynamic quantities should reproduce known Monte Carlo results. ${ }^{1}$ In the spirit of the Rosenfeld-Ashcroft ${ }^{3}$ universality principle, these values are then taken to be essentially unchanging for all potentials of the same symmetry; a similar treatment is made for the parameter $m$ introduced later in Eq. (3). In the RHNC-VM closure relation, the reference system bridge function $B_{0}(12)$ is then constructed with these diameters us- ing an approximation formulated for hard spheres by Verlet ${ }^{4}$ some years ago and later modified for homonuclear and heteronuclear hard diatomics by Labík et al. ${ }^{5}$

In the present work, continuing a progression towards more complex models that might consistently describe real fluids, we consider a dipolar fluid composed of heteronuclear diatomics with Lennard-Jones interatomic potentials and a point dipole at the molecular center of mass (2CLJD). In order to build directly into the reference system the molecular asymmetry introduced by the dipole, we reformulate the previous expression for the atomic diameters to explicitly incorporate the dipole moment $\mu$, now putting

$$
\begin{aligned}
d_{\alpha}= & \sigma_{\alpha} g(\gamma)\left(25-\ln \frac{k T}{\epsilon_{\alpha}\left(1-m \mu^{2} / \epsilon_{\alpha} \sigma_{\alpha}^{3}\right)}\right) \\
& \times\left[25+\ln \left(\rho \sigma_{\alpha}^{3}\right)\right], \quad \alpha=a, b
\end{aligned}
$$

where, as in Eq. (1), $\gamma=\sigma_{b} / \sigma_{a} \leqslant 1$. We complete the specification of the reference model by assigning to it the same molecular elongation as the "real" fluid, so that $L_{H D} / d_{a}=L / \sigma_{\alpha}=L^{*}$, where $L_{H D}$ and $L$ are the respective distances between atomic centers in the two systems.

The $g(\gamma)$ function, and more specifically the parameter $h$, reflects the differences in atomic diameters, while the parameter $m$, also adjustable, is associated with the dipole moment. We note that Eq. (3) is constructed so that if $\mu=0$ and $\sigma_{a}=\sigma_{b}(\gamma=1)$ the resulting theory is that of a nonpolar homonuclear fluid. ${ }^{6}$

In a recent publication on hydrogen chloride, ${ }^{7}$ we have studied a theoretical model with characteristics similar to those already described. The approximations used differ, however, in the treatment of the double molecular asymmetry: geometric, due to the difference in atomic diameters, and 
dipolar. In the present study these are separately and explicitly accounted for in the reference model through Eq. (3), while in the earlier work they are implicitly introduced through a single adjustable parameter, $\gamma$, whereby $d_{b}=\gamma d_{a}$, where

$$
d_{a}=\alpha \sigma_{a}\left[25-\ln \left(k T / \epsilon_{a}\right)\right]\left[25+\ln \left(\rho \sigma_{a}^{3}\right)\right] .
$$

The fundamental relations of the theory of liquids are the Ornstein-Zernike $(\mathrm{OZ})$ equation coupling the indirect and direct correlation functions, $\gamma(12)$ and $c(12)$,

$$
\gamma(12)=\frac{\rho}{4 \pi} \int c(13)[c(32)+\gamma(32)] d \mathbf{3},
$$

and the closure relation which, in the RHNC-VM approximation, is written as

$c(12)=\exp \left[-\beta u(12)+\gamma(12)-B_{0}(12)\right]-\gamma(12)-1$,

where $\beta=1 / k_{B} T, u(12)$ is the intermolecular potential, and $B_{0}(12)$ is the bridge function of the reference system described earlier. With the exception of Eq. (3), which replaces Eq. (8) of Ref. 1, further details on the use of the RHNC-VM approximation are as described in that earlier work on nonpolar heteronuclear systems, to which the reader is referred.

For completeness, we have also carried out systematic Monte Carlo (MC) simulations for the structural, thermodynamic, and dielectric properties of the models considered here. There appear to be in the literature no additional simulation studies for heteronuclear 2CLJD systems beyond the molecular dynamics calculations of Murad et al. ${ }^{8}$ for hydrogen chloride and the MC calculations carried out by us ${ }^{7,9}$ for the same system. Even for the simpler case of homonuclear 2CLJD models, available results known to us are limited to those of Fisher and co-workers ${ }^{10,11}$ for the refrigerant R152a $\left(\mathrm{CH}_{3}-\mathrm{CHF}_{2}\right)$, modeled as a fluid of this type.

The most significant electrostatic property of a material system is doubtless its dielectric constant, which measures the response of the system to an external electric field. It is however also the most problematical for computer simulation, in part due to the very long runs needed to obtain reliable values of this slowly-converging quantity. This may be one of the reasons for the scarcity of such data, especially for "realistic" models like those considered here. To help alleviate this deficiency, we have carried out high-precision, systematic simulations for the dielectric constant of our models, with runs of up to $450 \times 10^{6}$ configurations (depending on the thermodynamic conditions) to guarantee the stability of the computed values.

\section{POTENTIAL MODEL AND PARAMETER $\boldsymbol{m}$}

The intermolecular potential $u(12)$ of a heteronuclear 2CLJD system with the (ideal) molecular dipole aligned with the molecular axis is given by

$$
u\left(R \omega_{1} \omega_{2}\right)=u_{2 \mathrm{CLJ}}\left(R \omega_{1} \omega_{2}\right)+u_{D D}\left(R \omega_{1} \omega_{2}\right) .
$$

The first term in this expression is the two-center LennardJones (2CLJ) potential

$$
u_{2 \mathrm{CLJ}}\left(R \omega_{1} \omega_{2}\right)=\sum_{\alpha \beta} u_{\alpha \beta}(r), \quad \alpha, \beta=a, b,
$$

where

$$
u_{\alpha \beta}(r)=4 \epsilon_{\alpha \beta}\left[\left(\frac{\sigma_{\alpha \beta}}{r}\right)^{12}-\left(\frac{\sigma_{\alpha \beta}}{r}\right)^{6}\right]
$$

is the interaction between atoms $\alpha$ and $\beta$ of molecules 1 and 2 , respectively, separated by a distance $r=r\left(R \omega_{1} \omega_{2}\right)$. In these expressions, $R=\left|\mathbf{R}_{2}-\mathbf{R}_{1}\right|$ is the separation between molecular centers with positions $\mathbf{R}_{1}, \mathbf{R}_{2}$ and $\omega_{1}, \omega_{2}$ are the molecular orientations. For simplicity, we will write the Lennard-Jones parameters $\epsilon_{a a}, \epsilon_{b b}, \sigma_{a a}$, and $\sigma_{b b}$ as $\epsilon_{a}, \epsilon_{b}$, $\sigma_{a}$, and $\sigma_{b}$. The parameters $\epsilon_{a b}=\epsilon_{b a}$ and $\sigma_{a b}=\sigma_{b a}$ associated with the cross interactions are defined by the usual mixture rules,

$$
\begin{aligned}
& \epsilon_{a b}=\left(\epsilon_{a} \epsilon_{b}\right)^{1 / 2}, \\
& \sigma_{a b}=\frac{1}{2}\left(\sigma_{a}+\sigma_{b}\right) .
\end{aligned}
$$

The second term of Eq. (6) is the dipole-dipole interaction,

$$
u_{D D}\left(R \omega_{1} \omega_{2}\right)=-\frac{\mu^{2}}{R^{3}}\left[3\left(\mathbf{n} \cdot \mathbf{s}_{1}\right)\left(\mathbf{n} \cdot \mathbf{s}_{2}\right)-\mathbf{s}_{1} \cdot \mathbf{s}_{2}\right],
$$

where $\mathbf{n}$ is a unit vector in the direction of $\mathbf{R}=\mathbf{R}_{2}-\mathbf{R}_{1}$ and $\mathbf{s}_{1}, \mathbf{s}_{2}$ are unit vectors in the directions of the dipole moments of molecules 1 and 2, respectively.

The dipole-dipole interaction is notably long-ranged for computer simulations. To account for the long-range contributions we have adopted the reaction field (RF) method, ${ }^{12,13}$ which appears to be the most advantageous for establishing direct comparisons between theory and simulation. ${ }^{14}$ In this approximation scheme, the interaction potential $u(12)$ is truncated at a distance $R_{c}$, the cutoff radius, determined essentially by the size of the simulation cell. A given dipole $i$ of the sample interacts with its neighbors within the sphere of radius $R_{c}$ (centered on the dipole) according to the dipolar potential (11) and with the remainder, treated as an infinite, continuous, and polarizable dielectric medium of dielectric constant $\epsilon_{\mathrm{RF}}$, through a mean reaction field ${ }^{15,16}$

$$
\mathbf{R}_{i}=\frac{2\left(\epsilon_{\mathrm{RF}}-1\right)}{\left(2 \epsilon_{\mathrm{RF}}+1\right)} \frac{\mathbf{M}_{i}}{R_{c}^{3}},
$$

generated at the position of dipole $i$ by the polarization of the dielectric due to the total dipole moment $\mathbf{M}_{i}$ of the truncation sphere about $i$.

Neumann and Steinhauser ${ }^{15}$ have shown that the RF method is equivalent to using an effective potential given by ${ }^{13,15}$

$$
u_{e}(12)=\left\{\begin{array}{l}
u(12)+u_{\mathrm{RF}}(12) \quad R<R_{c} \\
0 \quad R>R_{c},
\end{array}\right.
$$

where $u_{\mathrm{RF}}(12)$ is the contribution of the interaction with the reaction field,

$$
u_{\mathrm{RF}}(12)=-\frac{2\left(\epsilon_{\mathrm{RF}}-1\right)}{\left(2 \epsilon_{\mathrm{RF}}+1\right)} \frac{\mu^{2}}{R_{c}^{3}}\left(\mathbf{s}_{1} \cdot \mathbf{s}_{2}\right) .
$$


TABLE I. Molecular parameters for the model fluids included in this work.

\begin{tabular}{ccc}
\hline \hline Parameter & Model $A$ & Model $B$ \\
\hline$\epsilon_{a} / k$ & $259.0 \mathrm{~K}$ & $259.0 \mathrm{~K}$ \\
$\epsilon_{b} / k$ & $20.0 \mathrm{~K}$ & $20.0 \mathrm{~K}$ \\
$\sigma_{a}$ & $3.353 \AA$ & $3.353 \AA$ \\
$\sigma_{b}$ & $2.735 \AA$ & $2.735 \AA$ \\
$\mu$ & $1.422 \mathrm{D}$ & $1.422 \mathrm{D}$ \\
$L$ & $1.257 \AA$ & $0.671 \AA$ \\
$\tau$ & 0.0284 & 0.0284 \\
\hline
\end{tabular}

To make theory and simulation directly comparable, ${ }^{14}$ all results in both methods have been obtained using the effective potential $u_{e}(12)$, with the same values of the parameters $R_{c}$ and $\epsilon_{\mathrm{RF}}$, rather than the original potential $u(12)$ given by Eqs. (6)-(11). The cutoff radius has been determined according to the standard relation $R_{c}=\frac{1}{2}(N / \rho)^{1 / 3}$, where $N$ is the number of molecules in the simulation sample.

We summarize in Table I the molecular parameters of the two (arbitrary) models, $A$ and $B$, for which we have carried out calculations. (In the table, $\tau=P_{b} / P_{a}$ is the ratio of atomic weights.) Most of the study focuses on model $A$, which has been chosen for theoretical consistency in fixing $m$ (see the following); it corresponds to the model of a nonpolar heteronuclear fluid used in Ref. 1 to determine the value of the parameter $h$ associated with the difference in atomic sizes. Although it is an arbitrary model, its LennardJones parameters, $\epsilon_{\alpha}$ and $\sigma_{\alpha}$, match those of a nonpolar system considered by Murad et al. ${ }^{8}$ as a model of liquid $\mathrm{HCl}$ in molecular dynamics studies.

Model $B$ differs from model $A$ only in having a smaller molecular anisotropy $\left(L^{*}=0.2\right)$, all other parameters being the same. This model is included in our study to accentuate the effects of the dipolar anisotropy over those of the geometric one, thereby permitting a stricter test of the capacity of the theoretical construction to deal specifically with the dipole-dipole interaction. The molecular geometries of the two models are contrasted in Fig. 1.

The reference system parameter $m$ of Eq. (3) remains to be fixed to complete the theoretical scheme of the RHNC-VM approximation. As with the other two parameters ( $\alpha$ and $h$ ) of Eq. (3) (see Ref. 1 and references therein), $m$ has been determined by requiring that, at a given temperature and density, the theoretical results for the compressibil-

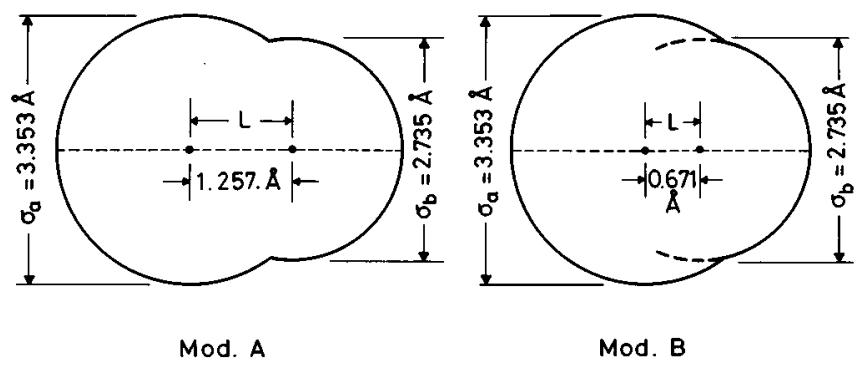

FIG. 1. Molecular geometries of models $A$ and $B$.
TABLE II. Theoretical compressibility factor and internal energy vs MC data at $T^{*}=0.8, \rho^{*}=0.75$, and several $m$ values for model $A$ of Table I.

\begin{tabular}{lccccc}
\hline \hline & \multicolumn{2}{c}{$\beta p / \rho$} & & \multicolumn{2}{c}{$\beta U / N$} \\
\cline { 2 - 3 } \cline { 5 - 6 }$m$ & MC & RHNC & & MC & RHNC \\
\hline 0.000 & -1.71 & -1.91 & & -11.18 & -11.14 \\
0.010 & -1.71 & -1.74 & & -11.18 & -11.13 \\
0.011 & -1.71 & -1.73 & & -11.18 & -11.12 \\
0.012 & -1.71 & -1.71 & & -11.18 & -11.12 \\
0.013 & -1.71 & -1.69 & & -11.18 & -11.12 \\
\hline \hline
\end{tabular}

ity factor and internal energy simultaneously match as closely as possible those of simulation. As noted earlier, we make the underlying assumption that these parameters are "universal" in that values determined for a particular molecular model should be equally valid for at least all other potentials of the same symmetry group. Thus, the new parameter $m$ has been adjusted while maintaining the previous values of $\alpha$ and $h$ obtained for $\mu=0$.

For reasons of consistency, we have chosen $m$ using the same molecular model as in Ref. 1 for the parameter $h$, to which we have simply added a reduced dipole moment $\mu^{* 2} \equiv \mu^{2} / \epsilon_{a} \sigma_{a}^{3}=1.50$, which corresponds to the dipole moment $\mu=1.422 D$ of Table I. The adjustment was carried out in both cases for the same state point, $T^{*}=0.8$ and $\rho^{*}=0.75$, lying in a region of low temperature and high density. In Table II we compare with MC the theoretical values of the compressibility factor and internal energy for different $m$, choosing

$$
m=1.2 \times 10^{-2}
$$

as the universal value for all calculations.

Table II also includes the value $m=0$. In this case the resultant expression for $d_{\alpha}$ is the one previously determined for a nonpolar heteronuclear fluid in Ref. 1, Eq. (1). The extent of the discrepancy seen here for the compressibility factor demonstrates the need for introducing the new parameter $m$ in extending the theory to polar systems.

\section{STRUCTURE AND THERMODYNAMICS}

In this section and in Sec. IV we compare theory with simulation. To facilitate that comparison, following Patey et al. ${ }^{13}$ we have computed all results in both cases using the effective potential $u_{e}(12)$, given by Eqs. (13) and (14), rather than the original potential $u(12)$.

For the numerical work, we have chosen the same reduced temperatures $\left(T^{*}=1.20\right.$ and 1.50) and reduced dipole moment $\left(\mu^{* 2}=1.50\right)$ for both model $A$ and model $B$. Since the two models also share the same Lennard-Jones parameters $\epsilon_{\alpha}$ and $\sigma_{\alpha}$ (Table I), this implies identical physical values of temperature $T$ and dipole moment $\mu$ in the two cases.

Table III gives the MC results for the compressibility factor $\beta p / \rho$, the internal energy $\beta U / N$, and the dipoledipole contribution to the energy $\beta U_{D D} / N$, for models $A$ and $B$ of Table I. In the simulations, we have applied different criteria for cases that did or did not include the dielectric constant. For the latter cases we have worked with 500 mol- 
TABLE III. Compressibility factor and internal energies from MC simulation.

\begin{tabular}{|c|c|c|c|c|c|c|c|}
\hline \multirow[b]{2}{*}{$T^{*}$} & \multirow[b]{2}{*}{$\rho^{*}$} & \multirow[b]{2}{*}{$\beta p / \rho$} & \multirow[b]{2}{*}{$\beta U / N$} & \multirow[b]{2}{*}{$\beta U_{D D} / N$} & \multirow[b]{2}{*}{ Sample } & \multicolumn{2}{|c|}{ No. configs. $\left(10^{6}\right)$} \\
\hline & & & & & & Equil. & Calc. \\
\hline & & & & Iodel $A$ & & & \\
\hline \multirow[t]{4}{*}{1.20} & 0.60 & -0.60 & -5.65 & -0.91 & 864 & 5 & 125 \\
\hline & 0.65 & -0.23 & -6.09 & -0.97 & 864 & 5 & 200 \\
\hline & 0.70 & 0.45 & -6.52 & -1.04 & 864 & 5 & 175 \\
\hline & 0.75 & 1.48 & -6.94 & -1.10 & 864 & 5 & 450 \\
\hline \multirow[t]{6}{*}{1.50} & 0.50 & -0.01 & -3.64 & -0.56 & 500 & 2 & 5 \\
\hline & 0.55 & 0.16 & -3.97 & -0.60 & 500 & 2 & 5 \\
\hline & 0.60 & 0.41 & -4.32 & -0.65 & 500 & 2 & 5 \\
\hline & 0.65 & 0.90 & -4.66 & -0.70 & 500 & 2 & 5 \\
\hline & 0.70 & 1.60 & -4.99 & -0.75 & 500 & 2 & 5 \\
\hline & 0.75 & 2.62 & -5.28 & -0.80 & 500 & 2 & 5 \\
\hline \multicolumn{8}{|c|}{ Model $B$} \\
\hline \multirow[t]{4}{*}{1.20} & 0.70 & -1.49 & -7.01 & -1.18 & 500 & 2 & 5 \\
\hline & 0.75 & -1.19 & -7.47 & -1.25 & 500 & 2 & 5 \\
\hline & 0.80 & -0.60 & -7.91 & -1.30 & 500 & 2 & 5 \\
\hline & 0.85 & 0.34 & -8.35 & -1.38 & 500 & 2 & 5 \\
\hline \multirow[t]{7}{*}{1.50} & 0.65 & -0.51 & -5.03 & -0.81 & 500 & 2 & 5 \\
\hline & 0.70 & -0.28 & -5.37 & -0.85 & 500 & 2 & 5 \\
\hline & 0.75 & 0.16 & -5.72 & -0.90 & 500 & 2 & 5 \\
\hline & 0.754 & 0.20 & -5.76 & -0.91 & 864 & 5 & 200 \\
\hline & 0.80 & 0.79 & -6.08 & -0.96 & 500 & 2 & 5 \\
\hline & 0.85 & 1.70 & -6.39 & -1.01 & 500 & 2 & 5 \\
\hline & 0.90 & 2.89 & -6.68 & -1.07 & 500 & 2 & 5 \\
\hline
\end{tabular}

ecules and runs of $2 \times 10^{6}$ configurations for equilibration and $5 \times 10^{6}$ more for data taking, while for the former cases we have used samples of 864 molecules with data-taking runs of $125 \times 10^{6}$ to $450 \times 10^{6}$ configurations following $5 \times 10^{6}$ of equilibration. Throughout this study we have used $\epsilon_{\mathrm{RF}}=7.20$ for the dielectric constant of the continuous medium, a value of the order of magnitude suggested by earlier exploratory runs.

Theory and simulation are graphically compared in Figs. $2-8$. For the structural properties, we have chosen as representative the results from the state point $T^{*}=1.20, \rho^{*}=0.70$ for model $A$ and $T^{*}=1.20, \rho^{*}=0.80$ for model $B$. In Fig. 2 we show the center-to-center distribution function $g_{000}$ [radial component of the molecular distribution function $g(12)]$ and in Fig. 3 the first three angular components $g_{k l m}$ of $g(12)$ in the axial frame of reference. In Figs. 2 and 3 the upper part corresponds to model $A$ and the lower to model $B$. Figure 4 shows a few higher-order angular components for model $A$ but not for model $B$. The latter case approximates a Stockmayer fluid (see Fig. 1) with components $g_{k l m}$ for $k>1$ that are almost inappreciable on the scale of Fig. 4. Finally, in Figs. 5 and 6 we show the theoretical curves obtained for the three different atom-atom pair distribution functions $g_{\alpha \beta}(r)$ for both models $A$ and $B$, respectively, contrasted with the corresponding MC points. The theoretical curves are obtained by direct integration over the molecular pair distribution function $g(12)$ following the method recently described in Ref. 17.

The theoretical results (solid line) and simulation (solid circles) for the thermodynamic properties are jointly presented in Fig. 7 for model $A$ and in Fig. 8 for model $B$. From

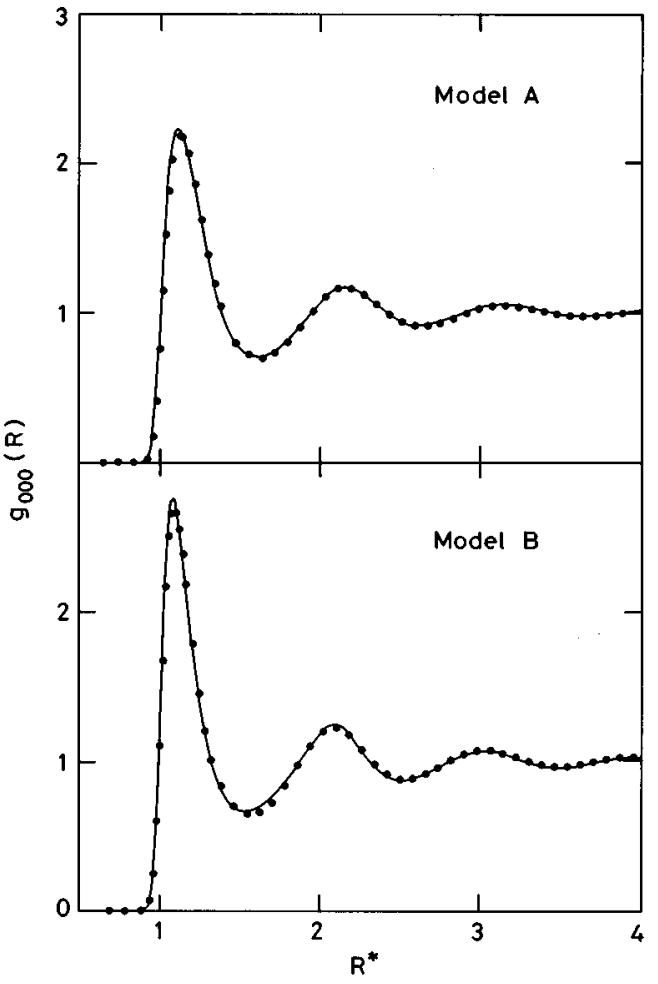

FIG. 2. Center-to-center distribution function $g_{000}(R)$ for model $A$ at $T^{*}=1.20$ and $\rho^{*}=0.70$ and for model $B$ at $T^{*}=1.20$ and $\rho^{*}=0.80$. Solid circles are $\mathrm{MC}$ values while solid lines represent theoretical results from the RHNC-VM equation. 


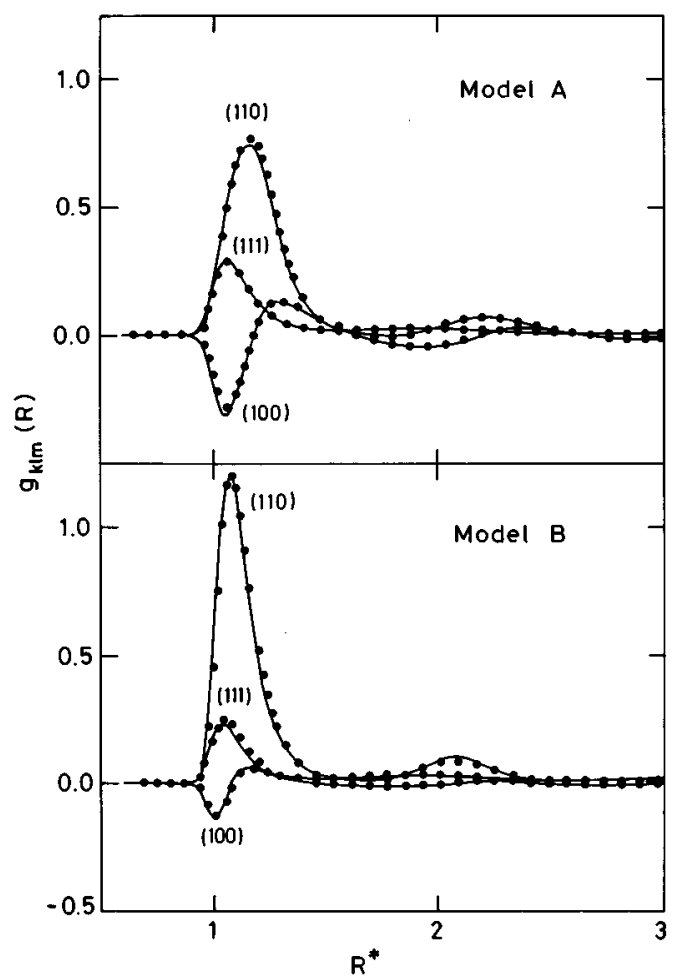

FIG. 3. First angular projections of the molecular distribution function $g$ (12) in the axial reference frame for model $A$ at $T^{*}=1.20$ and $\rho^{*}=0.70$ and for Model $B$ at $T^{*}=1.20$ and $\rho^{*}=0.80$. Symbols are as in Fig. 2 .

top to bottom these figures show (a) the compressibility factor $\beta p / \rho$, (b) the internal energy $\beta U / N$, and (c) the dipoledipole contribution to the energy $\beta U_{D D} / N$.

Direct inspection of all these figures shows that the agreement between theory and simulation is quite good, for both the structural (Figs. 2-6) as well as the thermodynamic (Figs. 7 and 8) properties. The level of agreement between the two techniques is furthermore similar for both model $A$ and model $B$.

The novelty of the RHNC-VM approximation we are examining lies in its generalization to include dipolar forces through an empirical relation between the geometry of the reference system and the dipole moment $\mu$ of the system of interest. Since the thermodynamic property most directly coupled to these forces is the dipole-dipole contribution to the energy, $\beta U_{D D} / N$, this quantity is particularly useful in checking the theory. In the Figs. 7(c) and 8(c) we see that the theoretical values lie systematically slightly above those of simulation, an apparent theoretical overestimation. Their relative deviations from the MC points, however, are everywhere less than 3\%; given the small magnitude of this quantity, the theoretical predictions may be taken as completely satisfactory. This is particularly significant for model $B$, since the small elongation of this case accentuates the dipolar effects compared to those of the structural anisotropy.

\section{DIELECTRIC CONSTANT}

The strong dependence on long-range forces of the dielectric constant $\epsilon$ makes its calculation by computer simu-

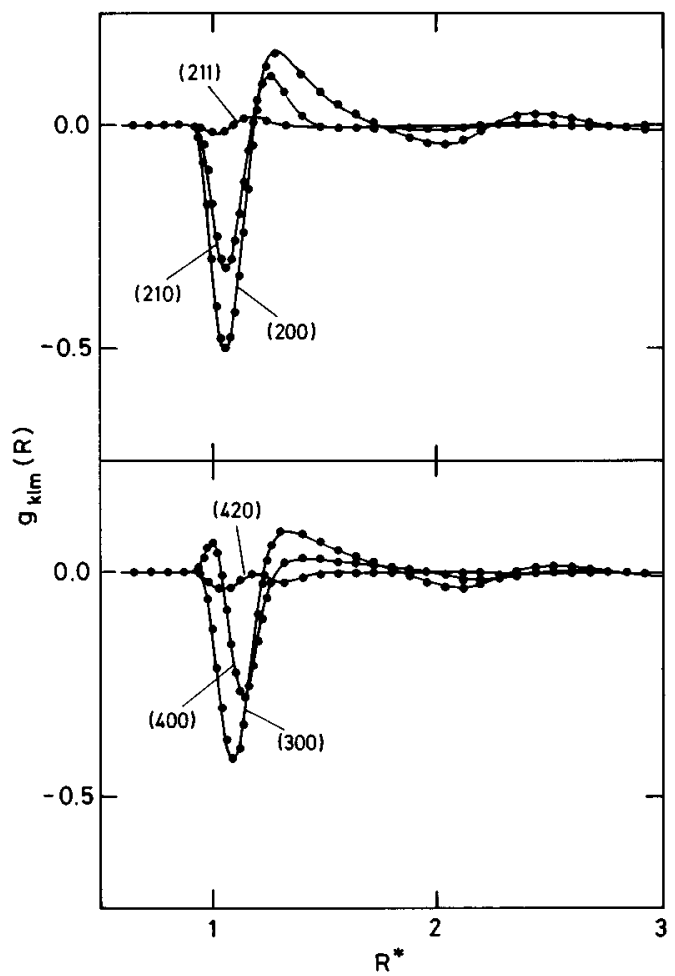

FIG. 4. Higher order angular projections of the molecular distribution function $g(12)$ in the axial reference frame for model $A$ at $T^{*}=1.20$ and $\rho^{*}=0.70$. Symbols are as in Fig. 2 .

lation, as well as comparisons between simulation and theory, problematical topics even today. Presentation of results of this nature must therefore carefully specify both the conditions of the simulation as well as the chosen procedure for properly comparing the two sets of results, theoretical and simulated.

For fluids of polar, nonpolarizable molecules, use of the RF boundary conditions leads to an $\epsilon$ satisfying ${ }^{13,18}$

$$
\frac{(\epsilon-1)\left(2 \epsilon_{\mathrm{RF}}+1\right)}{3\left(2 \epsilon_{\mathrm{RF}}+\epsilon\right)}=y g,
$$

where $y=4 \pi \beta \rho \mu^{2} / 9$ and $g$ is the Kirkwood factor. The computation of $g$ obviously will differ in the two approaches, simulation and theory.

If $N$ is the number of identical molecules in the cubic simulation cell and $\mathbf{M}=\Sigma \boldsymbol{\mu}_{k}(k=1, \ldots, N)$ is the total dipole moment of the cell, the $g$ factor is found from the mean square moment $\left\langle\mathbf{M}^{2}\right\rangle$ according to ${ }^{13,19,20}$

$$
g=\frac{\left\langle\mathbf{M}^{2}\right\rangle}{N \mu^{2}},
$$

where $\mu=\left|\boldsymbol{\mu}_{k}\right|$. Equations (16) and (17) jointly constitute the fluctuation formula for the dielectric constant $\epsilon$ to be used when, as here, RF boundary conditions are adopted in the simulation to correct for the long-range effects. It should be noted that this procedure implies that $\langle\mathbf{M}\rangle=0$; thus, as a simulation proceeds, fluctuations in $\mathbf{M}$ should evolve such 


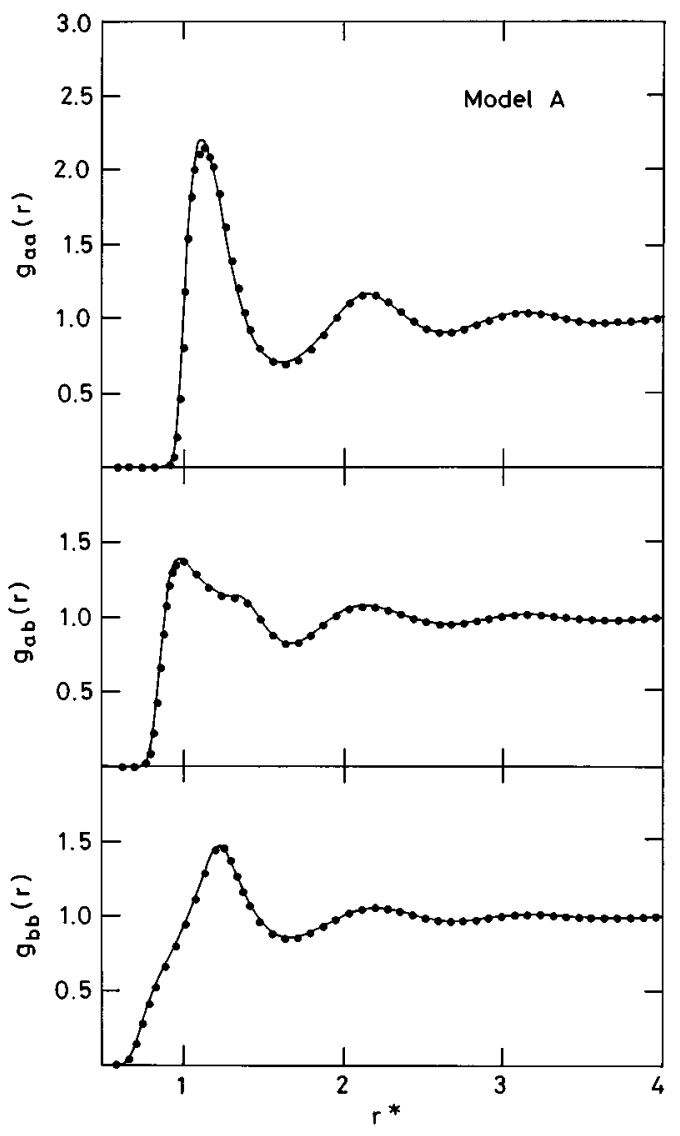

FIG. 5. Atom-atom pair distribution functions $g_{\alpha \beta}(r)$ for model $A$ at $T^{*}=1.20$ and $\rho^{*}=0.70$. Symbols are as in Fig. 2 .

that $\left(\left\langle\mathbf{M}^{2}\right\rangle-\langle\mathbf{M}\rangle^{2}\right) / N \mu^{2} \rightarrow\left\langle\mathbf{M}^{2}\right\rangle / N \mu^{2}$, a condition that must be met, within some specified margin of error, for reliable results.

When, as here, theoretical results for the dielectric constant of polar fluids are to be contrasted with those of simulation, the original Kirkwood formula ${ }^{21}$ for infinite systems is no longer valid. Patey et al. ${ }^{13}$ have shown that for comparison with simulations using RF boundary conditions, the $g$ factor is to be obtained instead from

$$
g=1+\frac{4 \pi \rho}{3} \int_{0}^{\infty} h_{\Delta}^{(e)}(R) R^{2} d R
$$

with $\epsilon$ again found using Eq. (16). In this expression, we have put $h_{\Delta}^{(e)}(R)=h_{110}^{(e)}(R)-2 h_{111}^{(e)}(R)$, where $h_{110}^{(e)}$ and $h_{111}^{(e)}$ are spherical harmonic coefficients of the total pair correlation function $h^{(e)}(12)$ in an axial frame where the $z$ axis coincides with the molecular axis. The superscript ' $e$ ' means that while the system is still infinite, the molecular interactions occur through the effective potential $u_{e}(12)$ defined by Eqs. (13) and (14) rather than the original potential $u(12)$.

Equation (18) can be written equivalently as

$$
g=1+\frac{1}{3} \rho \tilde{h}_{\Delta}^{(e)}(0)
$$

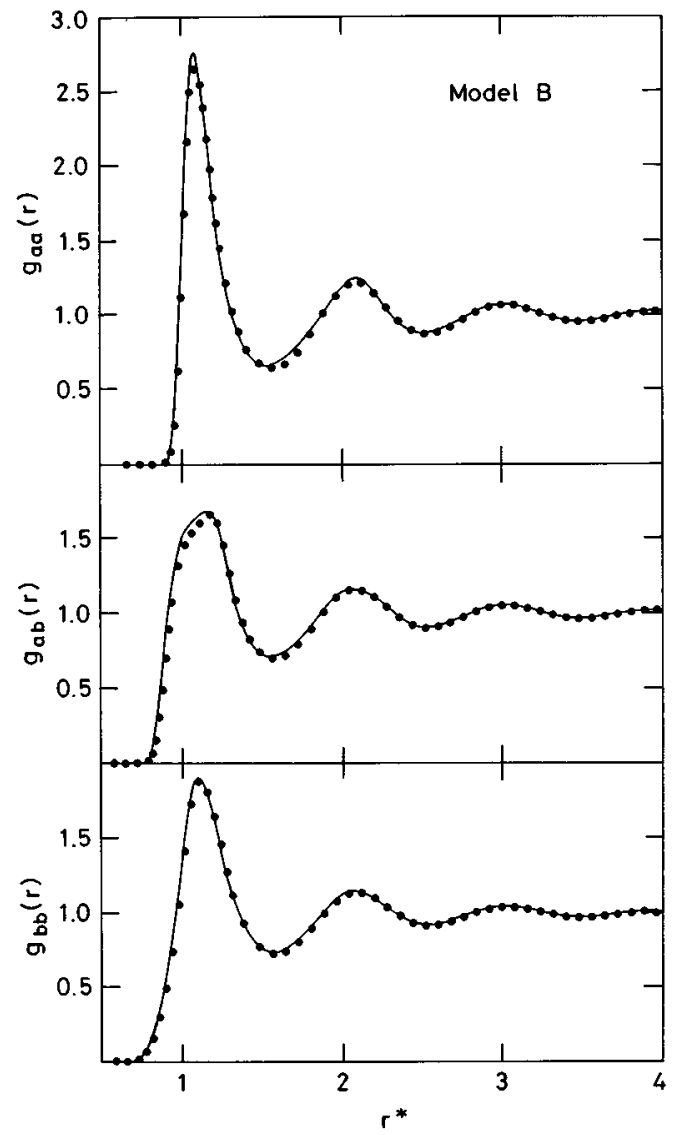

FIG. 6. Atom-atom pair distribution functions $g_{\alpha \beta}(r)$ for model $B$ at $T^{*}=1.20$ and $\rho^{*}=0.80$. Symbols are as in Fig. 2 .

where $\widetilde{h}_{\Delta}^{(e)}(0)$ is the Fourier transform of $h_{\Delta}^{(e)}(R)$ for $k=0$. Since $\widetilde{h}_{\Delta}^{(e)}(k)$ is computed in the process of solving the $\mathrm{OZ}$ integral equation, this latter form minimizes computational error and is to be preferred.

All results for $\epsilon$ presented here have been obtained using Eqs. (16) and (17) for MC simulation and Eqs. (16) and (19) for theory. The effective potential $u_{e}(12)$ has been used throughout, with a cutoff radius of $R_{c}=\frac{1}{2}(864 / \rho)^{1 / 3}$ determined by the $N=864$ particles in the MC simulations. These procedures should guarantee, in accord with the findings of Patey et al. ${ }^{13}$ that the two sets of values for $\epsilon$ may be directly compared.

The achievement of a high level of rigor and reliability in the simulations, within the inevitable limits imposed by available resources, has been a constant concern in this work. This has been particularly true in the calculation of $\epsilon$, for which, given its slow rate of convergence, runs of up to $450 \times 10^{6}$ configurations have been carried out. The cost in machine time that this entails has meant that simulations have focused primarily on model $A$, for which four density states have been simulated on the (low temperature) $T^{*}=1.20$ isotherm, a presumed good sampling of the liquid phase at this temperature. The dielectric constant for model $B$ has been simulated for just a single state, $T^{*}=1.50$ and $\rho^{*}=0.754$. This state has been chosen so that the density 


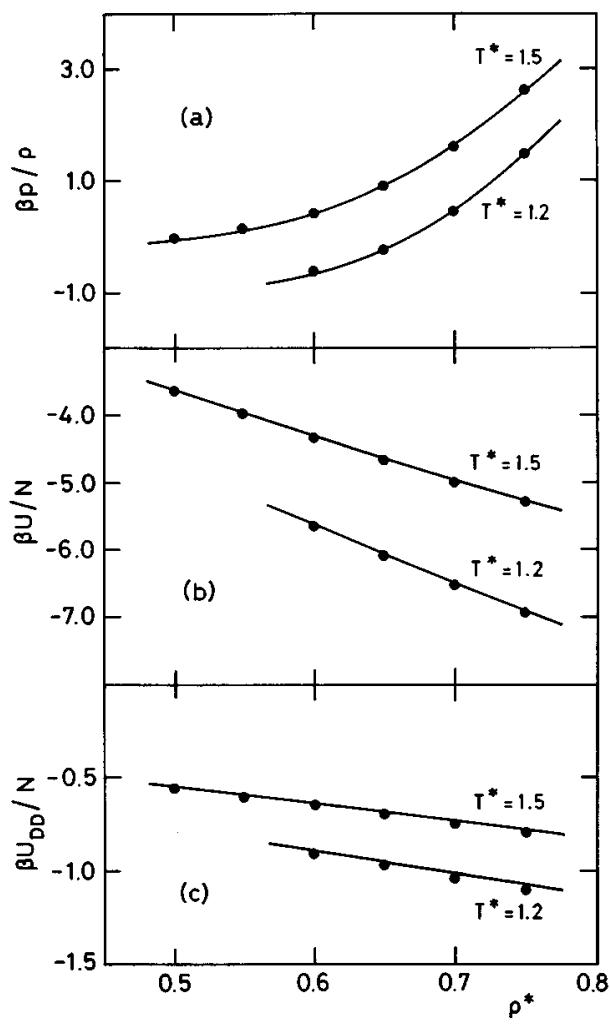

FIG. 7. (a) Compressibility factor, (b) internal energy, and (c) dipole-dipole contribution to the internal energy for model $A$, as functions of density. Dots and lines stand for MC and theoretical results, respectively.

$\rho_{d}^{*}=\rho d^{3}$, [where $d$ is the diameter of a sphere whose volume equals that of a hard diatomic molecule defined by the respective Lennard-Jones parameters $\sigma_{\alpha}$ (Ref. 1)] for this state of $B$ matches that of $\rho^{*}=0.65$ for $A$. Even if for just one state, the model $B$ calculation is of interest in that it tests the theory on a model different from that used to adjust the RHNC-VM reference system (see Sec. II). Since model $B$ additionally gives greater relative weight to the dipolar molecular asymmetry over the geometric, a comparison of the dielectric constants for this case is clearly an important test of the accuracy of the theory.

Table IV summarizes the MC results and includes the number of configurations generated for equilibration and calculation for each state. The evolution of $\epsilon$ with number of configurations is shown in Figs. 9 and 10 for model $A$ and model $B$ fluids, respectively, while Fig. 11 shows the evolution of the convergence condition $\left(\left\langle\mathbf{M}^{2}\right\rangle-\langle\mathbf{M}\rangle^{2}\right) / N \mu^{2}$ $\rightarrow\left\langle\mathbf{M}^{2}\right\rangle / N \mu^{2}$ during the course of the simulation for the state $T^{*}=1.20, \rho^{*}=0.70$ of model $A$. Other states simulated all display a degree of convergence equal to or better than that of Fig. 11 and are not shown.

Theoretical results for $\epsilon$ are compared to those of MC simulation in Fig. 12 for model $A$ and in Table V for model $B$. The first notable feature of the figure is the abnormally low value of the simulation result at the highest density, $\rho^{*}=0.75$. Assuming no shortcomings in the simulation, different explanations may be put forward for this behavior. At

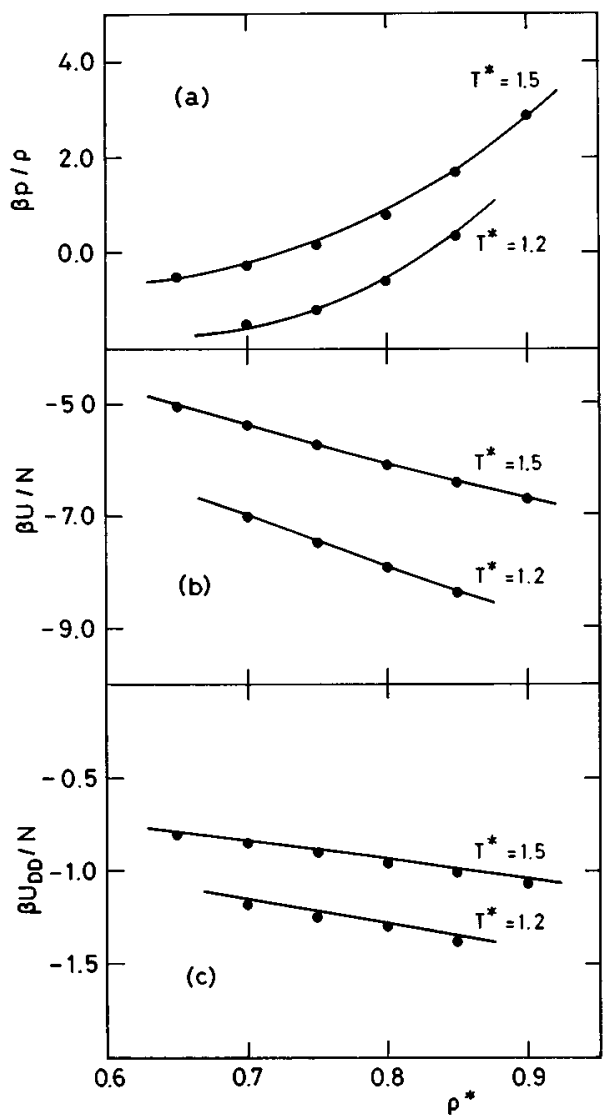

FIG. 8. (a) Compressibility factor, (b) internal energy, and (c) dipole-dipole contribution to the internal energy for model $B$, as functions of density. Dots and lines stand for MC and theoretical results, respectively.

these high densities, for one, we could be seeing the effect of density dielectric saturation due to the competition between short-range packing effects arising from the molecular anisotropy (form factor), on the one hand, and long-range dielectric effects, on the other. The predominance of the first of these over the second would reduce the capacity of the system to polarize in an external electric field, attenuating (or even inverting) the rate of growth of $\epsilon$ with $\rho^{*}$. If this view is correct, the theory is clearly failing, since it shows no tendency for $\epsilon$ to saturate. To test whether the phenomenon might show up at higher densities, we have continued the

TABLE IV. Dielectric constant from MC simulation of 864-particle samples at several densities.

\begin{tabular}{llcc}
\hline \hline & & \multicolumn{2}{c}{ No. configs. $\left(10^{6}\right)$} \\
\cline { 3 - 4 }$\rho^{*}$ & $\epsilon$ & Equil. & Calc. \\
\hline \multicolumn{4}{c}{ Model $A$ at $T^{*}=1.20$} \\
0.600 & 6.34 & 5 & 125 \\
0.650 & 6.96 & 5 & 200 \\
0.700 & 7.67 & 5 & 175 \\
0.750 & 7.70 & 5 & 450 \\
& & & \\
0.754 & 7.41 & 5 & 200 \\
\hline \hline
\end{tabular}



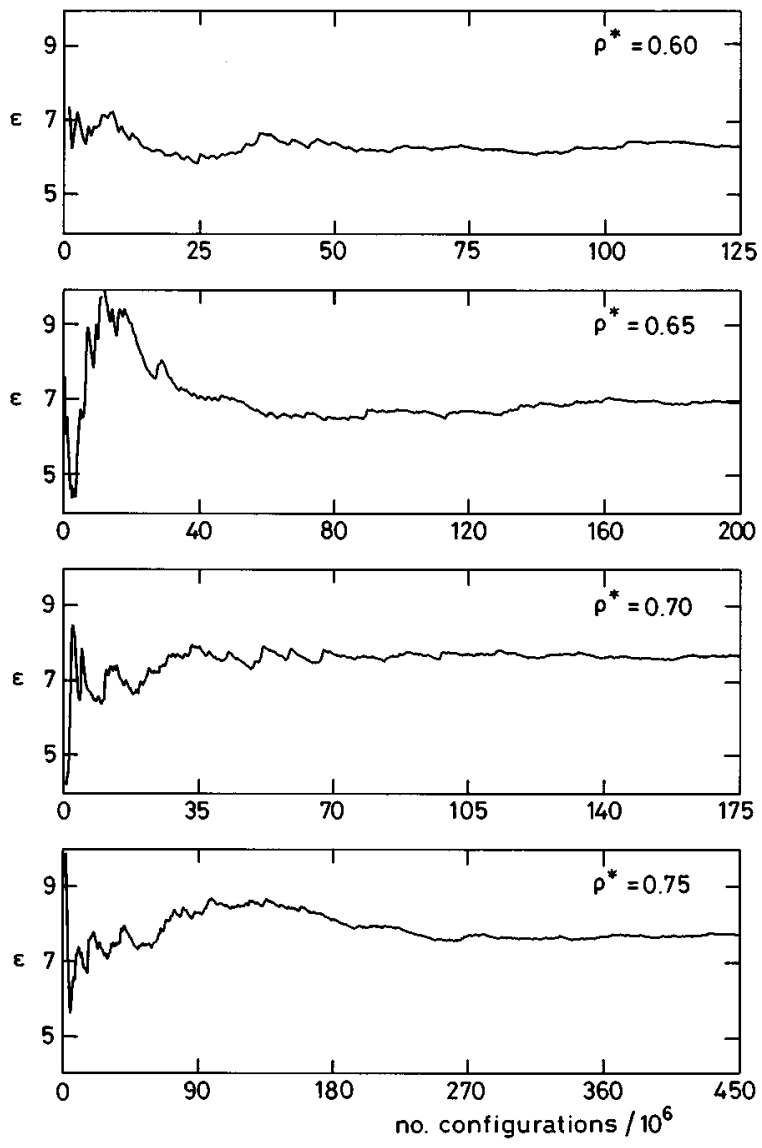

FIG. 9. Convergence of the MC dielectric constant along simulation runs. Results for model $A$ corresponding to the four state points of Table IV $\left(T^{*}=1.20\right)$.

theoretical solutions (not shown in Fig. 12) to a density of $\rho^{*}=0.90$, with no change in the nature of the theoretical curve. However, we note that some time ago, Nichols and Calef ${ }^{22}$ found that an RHNC scheme could produce dielectric saturation at sufficiently high densities. They suggest that this result, obtained for a model of hard linear triatomics with a hard sphere fluid as reference system, might result from an excessively large diameter of the reference hard spheres. This idea could not be pursued in the present calculation, which uses a different reference system.

An alternative explanation could be that the low value of the simulated $\epsilon$ at high densities is an artifact of an insuffi-

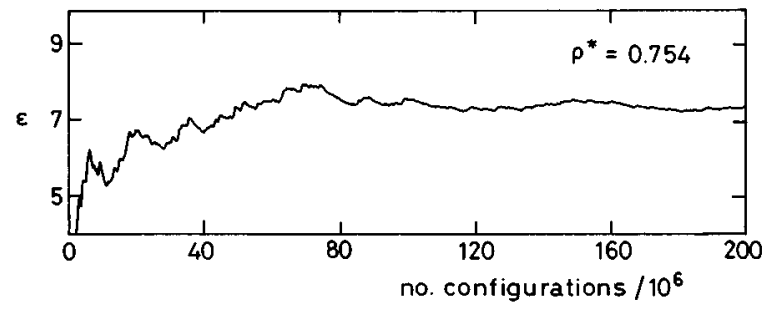

FIG. 10. Convergence of the MC dielectric constant along the simulation run. Results for model $B$ at $T^{*}=1.50$.

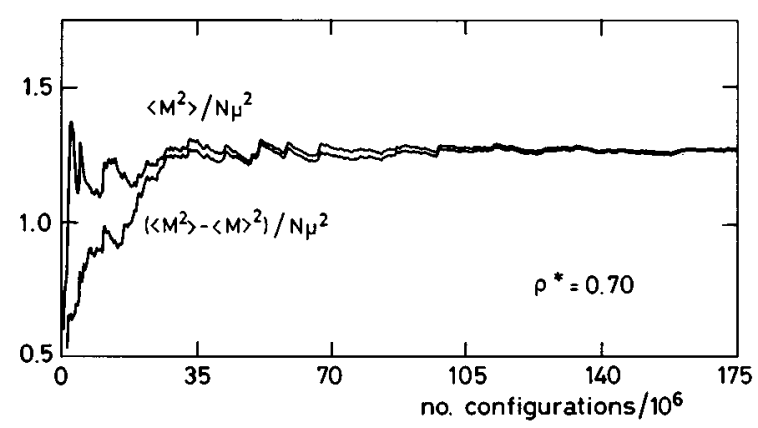

FIG. 11. Evolution of the fluctuation $\left(\left\langle\mathbf{M}^{2}\right\rangle-\langle\mathbf{M}\rangle^{2}\right) / \mathbf{N} \mu^{2}$ and of the average $\left\langle\mathbf{M}^{2}\right\rangle / \mathbf{N} \mu^{2}$ along the simulation run of model $A$ at the state point $T^{*}=1.20$ and $\rho^{*}=0.70$.

cient simulation sample size (864 particles), whereby longrange behavior that strongly affects $\epsilon$ cannot adequately be reproduced at sufficiently high densities. In such circumstances, the small sample size and correspondingly small cutoff radius $R_{c}$ would no longer guarantee the equivalence conditions between theory and simulation implied by Eqs. (16)-(18).

In contrast with the discrepancy between theory and experiment at high densities, the results for $\epsilon$ in a broad range of normal densities covered in the study of model $A$ (Fig. 12) can be characterized as very good. This is also true for the single state of model $B$, as seen in Table V. Given the delicacy and complexity of calculations for the dielectric constant, both theoretical and simulated, this must be counted as a notable success for the RHNC-VM theory.

\section{DISCUSSION}

We have carried out extensive Monte Carlo simulations for two models of fluids of dipolar heteronuclear two-center Lennard-Jones molecules using reaction field boundary conditions. The study included structural, thermodynamic, and dielectric properties and their variation with density along two different isotherms for each model. A primary goal of this work has been to furnish simulation data of high preci-

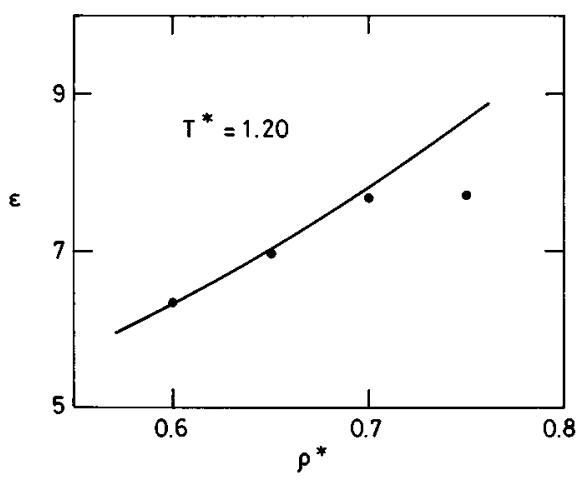

FIG. 12. Dielectric constant as a function of density for model $A$ at $T^{*}=1.20$. The solid line represents theoretical results and solid circles stand for MC simulations. 
TABLE V. Theoretical and MC dielectric constant $\epsilon$ for model $B$.

\begin{tabular}{lccc}
\hline \hline$T^{*}$ & $\rho^{*}$ & Theory & MC \\
\hline 1.50 & 0.754 & 7.57 & 7.41 \\
\hline \hline
\end{tabular}

sion for this class of fluids, towards which special efforts have been made to assure the best possible conditions of sample size, length of runs, etc., especially for the dielectric constant.

We have also studied in this work an RHNC integral equation approximation which generalizes to this class of fluids a closure relation recently formulated for nonpolar fluids with excellent results. The reference system in this approximation is a fluid of hard heteronuclear diatomics whose atomic diameters depend empirically on the temperature and density of the system of interest and in the present version also on its molecular dipole moment $\mu$.

A comparison of the theoretical results with those from simulation completes this work: we find a high degree of agreement between the two, not only for the structural and thermodynamic properties but also for the more sensitive dielectric constant. The only significant discrepancy shows up for just this latter property at very high densities. We have no definitive explanation for this disagreement, which may arise from dielectric saturation or (what seems to us more likely) insufficient sample size at these high densities to properly account for long-range effects due to the dipolar potential.

The overall good results obtained from the theory for the set of properties and dipolar fluid models examined here confirms and extends the excellent behavior of the RHNC-VM approximation that was already established in earlier studies of the three possible types of nonpolar diatomic fluids (homonuclear diatomics, quadrupolar homonuclear diatomics, and nonpolar heteronuclear diatomics), in a region of the phase diagram that in each case covers the presumably essential part of the liquid state (see Ref. 1 and references therein). We may thus conclude in a general sense that this RHNC scheme, based on a closure relation we have denoted RHNC-VM, is a good approximation for diatomic fluids, polar and nonpolar. This further supports the assumed universality of the parameters $\alpha, h$, and $m$ that determine the reference atomic diameters $d_{\alpha}$, in the sense that they are essentially unchanging for all potentials of the same symmetry group, reflecting thereby the universality of the reference bridge function.

\section{ACKNOWLEDGMENTS}

We thank the Centro Técnico de Informática del CSIC and especially the Centro de Supercomputación de Galicia (CESGA) for generous access to their computing facilities. This work was financially supported by the Spanish Dirección General de Investigación Científica y Ténica (DGICYT) under Grant No. PB94-0112.

${ }^{1}$ M. Lombardero, C. Martín, and E. Lomba, Mol. Phys. 81, 1313 (1994).

${ }^{2}$ It should be noted that this value of the parameter $h$ differs from the value used in Ref. $1, h=0.89$. This difference occurs because in solving the OZ equation in the earlier work we assumed for greater numerical efficiency a minimum distance $r_{m}^{*}=0.7$ such that $u_{\alpha \beta}(r)=\infty$ for $r^{*} \leqslant r_{m}^{*}$. In the present work this unneeded condition has been dropped, thereby changing the calculated value of $h$ to 0.98 , as cited and used in this paper. We have also recalculated with this new value of $h$ all theoretical results of Ref. 1 . We find that the final results are scarcely affected by the change, save for those instances where appreciable differences existed between theory and simulation [as in the compressibility factor (Fig. 5 of Ref. 1) or the spherical harmonic coefficients $g_{k 10}$ (Fig. 4 of Ref. 1) of fluid $B$ ], which are now diminished in good measure. Thus, for example, the compressibility factor for the thermodynamic state with greatest error, $T^{*}=1.0, \rho^{*}=0.75$ (Fig. 5 of Ref. 1) goes from a value of 1.02 to a corrected value of 0.87 , compared with the MC value of 0.64 . The discrepancies affecting the $g_{k 10}$ functions are practically eliminated, so that the theoretical curves now show the same good agreement with the MC results as do the other $g_{k l m}$ coefficients (see Figs. 1-4 of Ref. 1). The latter good agreement remains.

${ }^{3}$ Y. Rosenfeld and N. W. Ashcroft, Phys. Rev. A 20, 1208 (1979).

${ }^{4}$ L. Verlet, Mol. Phys. 41, 183 (1980).

${ }^{5}$ S. Labík, A. Malijevský, and W. R. Smith, Mol. Phys. 73, 87 (1991); 73, 495 (1991).

${ }^{6}$ M. Lombardero, C. Martín, and E. Lomba, J. Chem. Phys. 97, 2724 (1992).

${ }^{7}$ E. Lomba, C. Martín, M. Lombardero, F. Lado, and J. S. Høye, J. Chem. Phys. 100, 1599 (1994).

${ }^{8}$ S. Murad, K. E. Gubbins, and J. G. Powles, Mol. Phys. 40, 253 (1980).

${ }^{9}$ E. Lomba, A. Fernández, C. Martín, and M. Lombardero, Phys. Scr. T38, 79 (1991).

${ }^{10}$ C. Vega, B. Saager, and J. Fischer, Mol. Phys. 68, 1079 (1989).

${ }^{11}$ B. Saager, J. Fischer, and M. Neumann, Mol. Simul. 6, 27 (1991).

${ }^{12}$ J. A. Barker and R. O. Watts, Chem. Phys. Lett. 3, 144 (1969); Mol. Phys. 26, 789 (1973).

${ }^{13}$ G. N. Patey, D. Levesque, and J. J. Weis, Mol. Phys. 45, 733 (1982).

${ }^{14}$ J. M. Caillol, D. Levesque, and J. J. Weis, Mol. Phys. 44, 733 (1981).

${ }^{15}$ M. Neumann and O. Steinhauser, Mol. Phys. 39, 437 (1980).

${ }^{16}$ J. A. Barker, Mol. Phys. 83, 1057 (1994).

${ }^{17}$ M. Alvarez, E. Lomba, C. Martín, and M. Lombardero, J. Chem. Phys. 103, 3680 (1995)

${ }^{18}$ M. Newmann, Mol. Phys. 50, 841 (1983).

${ }^{19}$ S. W. Leeuw, J. W. Perram, and E. R. Smith, Annu. Rev. Phys. Chem. 37, 245 (1986).

${ }^{20}$ P. G. Kusalik, Mol. Phys. 81, 199 (1994).

${ }^{21}$ J.-P. Hansen and I. R. McDonald, Theory of Simple Liquids (Academic, London, 1986).

${ }^{22}$ A. L. Nichols III and D. F. Calef, Mol. Phys. 71, 269 (1990). 